\title{
EXISTENCE OF SOLUTIONS FOR NONLINEAR IMPULSIVE DYNAMIC EQUATIONS ON A TIME SCALE
}

\author{
Abdelouaheb Ardjouni, Ahcene Djoudi
}

\begin{abstract}
Let $\mathbb{T}$ be a time scale such that $0, t_{i}, T \in \mathbb{T}, i=1,2, \ldots, n$, and $0<t_{i}<t_{i+1}$. Assume each $t_{i}$ is dense. Using a fixed point theorem due to Krasnoselskii-Burton, we show that the nonlinear impulsive dynamic equation

$$
\left\{\begin{array}{l}
y^{\Delta}(t)=-a(t) h\left(y^{\sigma}(t)\right)+f(t, y(t)), t \in(0, T] \\
y(0)=0 \\
y\left(t_{i}^{+}\right)=y\left(t_{i}^{-}\right)+I\left(t_{i}, y\left(t_{i}\right)\right), i=1,2, \ldots, n
\end{array}\right.
$$

where $y\left(t_{i}^{ \pm}\right)=\lim _{t \rightarrow t_{i}^{ \pm}} y(t)$, and $y^{\Delta}$ is the $\Delta$-derivative on $\mathbb{T}$, has a solution.

Keywords: Fixed point, large contraction, time scales, nonlinear impulsive dynamic equations.
\end{abstract}

\section{Introduction}

Let $\mathbb{T}$ be a time scale such that $0, t_{i}, T \in \mathbb{T}$, for $i=1,2, \ldots, n, 0<t_{i}<t_{i+1}$, and assume that $t_{i}$ is dense in $\mathbb{T}$ for each $i=1,2, \ldots, n$. In this paper, we are interested in the analysis of qualitative theory of solutions to impulsive dynamic equations. Motivated by the papers [1]-[4], [8]-[15], [19] and the references therein, we consider the following totally nonlinear impulsive dynamic equation

$$
\left\{\begin{array}{l}
y^{\Delta}(t)=-a(t) h\left(y^{\sigma}(t)\right)+f(t, y(t)), t \in(0, T], \\
y(0)=0, \\
y\left(t_{i}^{+}\right)=y\left(t_{i}^{-}\right)+I\left(t_{i}, y\left(t_{i}\right)\right), i=1,2, \ldots, n,
\end{array}\right.
$$

where $y\left(t_{i}^{ \pm}\right)=\lim _{t \rightarrow t_{i}^{ \pm}} y(t), y\left(t_{i}\right)=y\left(t_{i}^{-}\right), a: \mathbb{T} \rightarrow \mathbb{R}$ is a positive rd-continuous real-valued function, $h: \mathbb{R} \rightarrow \mathbb{R}$ is continuous function, $f, I: \mathbb{T} \times \mathbb{R} \rightarrow \mathbb{R}$ satisfying the Lipschitz condition, $[0, T]=\{t \in \mathbb{T}: 0 \leq t \leq T\}$. Note that the intervals $[\alpha, \beta)$, $(\alpha, \beta]$ and $(\alpha, \beta)$ are defined similarly.

Received May 11, 2017; accepted February 22, 2018.

2010 Mathematics Subject Classification. Primary 34K13; Secondary 34A34, 34K30, 34L30. 
In 1988, Stephan Hilger [12] introduced the theory of time scales (measure chains) as a means of unifying discrete and continuum calculi. Since Hilger's initial work there has been significant growth in the theory of dynamic equations on time scales, covering a variety of different problems; see $[6,7]$ and references therein. The study of impulsive initial and boundary value problems is extensive. For the theory and classical results, we direct the reader to the monographs $[5,16,17]$.

Our purpose here is to use a modification of Krasnoselskii's fixed point theorem due to Burton (see [8] Theorem 3) to show the existence of solutions on time scales for equation (1.1). Clearly, the present problem is totally nonlinear so that the variation of parameters can not be applied directly. Then, we resort to the idea of adding and subtracting a linear term. As noted by Burton in [8], the added term destroys a contraction already present in part of the equation but it replaces it with the so called a large contraction mapping which is suitable for fixed point theory. During the process we have to transform (1.1) into an integral equation written as a sum of two mapping; one is a large contraction and the other is compact. After that, we use a variant of Krasnoselskii fixed point theorem, to show the existence of a solution for equation (1.1).

In Section 2, we present some preliminary material that we will need through the remainder of the paper. We will state some facts about the exponential function on a time scale as well as the modification of Krasnoselskii's fixed point theorem established by Burton (see ([8] Theorem 3) and [9]). For details on Krasnoselskii's theorem we refer the reader to [18]. We present our main results in Section 3.

\section{Preliminaries}

The reader can find more details on the materials and basic properties used here in the first chapter of Bohner and Peterson book [6] and can find good examples on dynamic equations in Chapter 2 of [7].

A time scale $\mathbb{T}$ is a closed nonempty subset of $\mathbb{R}$. For $t \in \mathbb{T}$ the forward jump operator $\sigma$, and the backward jump operator $\rho$, respectively, are defined as $\sigma(t)=$ inf $\{s \in \mathbb{T}: s>t\}$ and $\rho(t)=\sup \{t \in \mathbb{T}: s<t\}$. These operators allow elements in the time scale to be classified as follows. We say $t$ is right scattered if $\sigma(t)>t$ and right dense if $\sigma(t)=t$. We say $t$ is left scattered if $\rho(t)<t$ and left dense if $\rho(t)=t$. The graininess function $\mu: \mathbb{T} \rightarrow[0, \infty)$, is defined by $\mu(t)=\sigma(t)-t$ and gives the distance between an element and its successor. We set inf $\varnothing=\sup \mathbb{T}$ and $\sup \varnothing=\inf \mathbb{T}$. If $\mathbb{T}$ has a left scattered maximum $M$, we define $\mathbb{T}^{k}=\mathbb{T} \backslash\{M\}$. Otherwise, we define $\mathbb{T}^{k}=\mathbb{T}$. If $\mathbb{T}$ has a right scattered minimum $m$, we define $\mathbb{T}_{k}=\mathbb{T} \backslash\{m\}$. Otherwise, we define $\mathbb{T}_{k}=\mathbb{T}$.

Let $t \in \mathbb{T}^{k}$ and let $f: \mathbb{T} \rightarrow \mathbb{R}$. The delta derivative of $f(t)$, denoted $f^{\triangle}(t)$, is defined to be the number (when it exists), with the property that, for each $\epsilon>0$, there is a neighborhood $U$ of $t$ such that

$$
\left|f(\sigma(t))-f(s)-f^{\triangle}(t)[\sigma(t)-s]\right| \leq \epsilon|\sigma(t)-s|,
$$


for all $s \in U$. If $\mathbb{T}=\mathbb{R}$ then $f^{\triangle}(t)=f^{\prime}(t)$ is the usual derivative. If $\mathbb{T}=\mathbb{Z}$ then $f^{\triangle}(t)=\triangle f(t)=f(t+1)-f(t)$ is the forward difference of $f$ at $t$.

A function $f$ is right dense continuous (rd-continuous), $f \in C_{r d}=C_{r d}(\mathbb{T}, \mathbb{R})$, if it is continuous at every right dense point $t \in \mathbb{T}$ and its left-hand limits exist at each left dense point $t \in \mathbb{T}$. The function $f: \mathbb{T} \rightarrow \mathbb{R}$ is differentiable on $\mathbb{T}^{k}$ provided $f^{\triangle}(t)$ exists for all $t \in \mathbb{T}^{k} . f \in C_{r d}^{1}=C_{r d}^{1}(\mathbb{T}, \mathbb{R})$ if $f^{\triangle} \in C_{r d}(\mathbb{T}, \mathbb{R})$.

We are now ready to state some properties of the delta-derivative of $f$. Note $f^{\sigma}(t)=f(\sigma(t))$.

Theorem 2.1. ([6, Theorem 1.20]) Assume $f, g: \mathbb{T} \rightarrow \mathbb{R}$ are differentiable at $t \in \mathbb{T}^{k}$ and let $\alpha$ be a scalar.

(i) $(f+g)^{\triangle}(t)=g^{\triangle}(t)+f^{\triangle}(t)$.

(ii) $(\alpha f)^{\triangle}(t)=\alpha f^{\triangle}(t)$.

(iii) The product rules

$$
\begin{aligned}
& (f g)^{\triangle}(t)=f^{\triangle}(t) g(t)+f^{\sigma}(t) g^{\triangle}(t), \\
& (f g)^{\triangle}(t)=f(t) g^{\triangle}(t)+f^{\triangle}(t) g^{\sigma}(t) .
\end{aligned}
$$

(iv) If $g(t) g^{\sigma}(t) \neq 0$ then

$$
\left(\frac{f}{g}\right)^{\triangle}(t)=\frac{f^{\triangle}(t) g(t)-f(t) g^{\triangle}(t)}{g(t) g^{\sigma}(t)} .
$$

The next theorem is the chain rule on time scales ([6, Theorem 1.93]).

Theorem 2.2. (Chain Rule) Assume $\nu: \mathbb{T} \rightarrow \mathbb{R}$ is strictly increasing and $\widetilde{\mathbb{T}}:=$ $\nu(\mathbb{T})$ is a time scale. Let $\omega: \widetilde{\mathbb{T}} \rightarrow \mathbb{R}$. If $\nu^{\triangle}(t)$ and $\omega^{\widetilde{\triangle}}(\nu(t))$ exist for $t \in \mathbb{T}^{k}$, then $(\omega \circ \nu)^{\triangle}=\left(\omega^{\widetilde{\Delta}} \circ \nu\right) \nu^{\triangle}$.

In the sequel we will need to differentiate and integrate functions of the form $f(t-\tau(t))=f(\nu(t))$ where, $\nu(t):=t-\tau(t)$. Our next theorem is the substitution rule $([6$, Theorem 1.98]).

Theorem 2.3. (Substitution) Assume $\nu: \mathbb{T} \rightarrow \mathbb{R}$ is strictly increasing and $\widetilde{\mathbb{T}}:=$ $\nu(T)$ is a time scale. If $f: \mathbb{T} \rightarrow \mathbb{R}$ is rd-continuous function and $\nu$ is differentiable with $r d$-continuous derivative, then for $a, b \in T$,

$$
\int_{a}^{b} f(t) \nu^{\triangle}(t) \triangle t=\int_{\nu(a)}^{\nu(b)}\left(f \circ \nu^{-1}\right)(s) \widetilde{\triangle} s
$$

A function $p: \mathbb{T} \rightarrow \mathbb{R}$ is said to be regressive provided $1+\mu(t) p(t) \neq 0$ for all $t \in \mathbb{T}^{k}$. The set of all regressive rd-continuous function $f: \mathbb{T} \rightarrow \mathbb{R}$ is denoted by $\mathcal{R}$ while the set $\mathcal{R}^{+}=\{f \in \mathcal{R}: 1+\mu(t) f(t)>0$ for all $t \in \mathbb{T}\}$. 
Let $p \in \mathcal{R}$ and $\mu(t) \neq 0$ for all $t \in \mathbb{T}$. The exponential function on $\mathbb{T}$ is defined by

$$
e_{p}(t, s)=\exp \left(\int_{s}^{t} \frac{1}{\mu(z)} \log (1+\mu(z) p(z)) \triangle z\right) .
$$

It is well known that if $p \in \mathcal{R}^{+}$, then $e_{p}(t, s)>0$ for all $t \in \mathbb{T}$. Also, the exponential function $y(t)=e_{p}(t, s)$ is the solution to the initial value problem $y^{\triangle}=p(t) y$, $y(s)=1$. Other properties of the exponential function are given in the following lemma.

Lemma 2.1. ([6]) Let $p, q \in \mathcal{R}$. Then

(i) $e_{0}(t, s)=1$ and $e_{p}(t, t)=1$;

(ii) $e_{p}(\sigma(t), s)=(1+\mu(t) p(t)) e_{p}(t, s)$;

(iii) $\frac{1}{e_{p}(t, s)}=e_{\ominus p}(t, s)$, where $\ominus p(t)=-\frac{p(t)}{1+\mu(t) p(t)}$;

(iv) $e_{p}(t, s)=\frac{1}{e_{p}(s, t)}=e_{\ominus p}(s, t)$

(v) $e_{p}(t, s) e_{p}(s, r)=e_{p}(t, r)$;

(vi) $e_{p}^{\triangle}(., s)=p e_{p}(., s)$ and $\left(\frac{1}{e_{p}(., s)}\right)^{\triangle}=-\frac{p(t)}{e_{p}^{\sigma}(., s)}$.

Lemma 2.2. ([1]) If $p \in \mathcal{R}^{+}$, then

$$
0<e_{p}(t, s) \leq \exp \left(\int_{s}^{t} p(u) \Delta u\right), \forall t \in \mathbb{T} .
$$

Corollary 2.1. ([1]) If $p \in \mathcal{R}^{+}$and $p(t)<0$ for all $t \in \mathbb{T}$, then for all $s \in \mathbb{T}$ with $s \leq t$ we have

$$
0<e_{p}(t, s) \leq \exp \left(\int_{s}^{t} p(u) \triangle u\right)<1 .
$$

Krasnoselskii (see [8] or [18]) combined the contraction mapping theorem and Schauder's theorem and formulated the following hybrid and attractive result.

Theorem 2.4. Let $M$ be a closed convex nonempty subset of a Banach space $(S,\|\|$.$) . Suppose that A$ and $B$ map $M$ into $S$ such that

(i) $\forall x, y \in M \Rightarrow A x+B y \in M$,

(ii) $A$ is continuous and $A M$ is contained in a compact set,

(iii) $B$ is a contraction with constant $\alpha<1$.

Then there is a $z \in M$ with $z=A z+B z$.

This is a captivating result and has a number of interesting applications. In recent year much attention has been paid to this theorem. Burton [8] observed that Krasnoselskii result can be more interesting in applications with certain changes and formulated in Theorem 2.6 below (see [8] for the proof). 
Definition 2.1. ([8]) Let $(M, d)$ be a metric space and $B: M \rightarrow M . B$ is said to be a large contraction if $\varphi, \psi \in M$, with $\varphi \neq \psi$ then $d(B \varphi, B \psi)<d(\varphi, \psi)$ and if for all $\varepsilon>0$ there exists $\delta<1$ such that

$$
[\varphi, \psi \in M, d(\varphi, \psi) \geq \varepsilon] \Rightarrow d(B \varphi, B \psi) \leq \delta d(\varphi, \psi)
$$

Theorem 2.5. ([8]) Let $(M, d)$ be a complete metric space and $B$ be a large contraction. Suppose there is an $x \in M$ and $L>0$, such that $d\left(x, B^{n} x\right) \leq L$ for all $n \geq 1$. Then $B$ has a unique fixed point in $M$.

Theorem 2.6. (Krasnoselskii-Burton) Let $M$ be a closed bounded convex nonempty subset of a Banach space $(S,\|\|$.$) . Suppose that A, B$ map $M$ into $M$ and that

(i) $\forall x, y \in M \Rightarrow A x+B y \in M$,

(ii) $A$ is continuous and $A M$ is contained in a compact subset of $M$,

(iii) $B$ is a large contraction.

Then there is a $z \in M$ with $z=A z+B z$.

It is obvious that if we want to apply the above theorem we need to construct two mappings, one is large contraction and the other is compact.

\section{Existence of solutions}

We will state and prove our main results in this section.

Define $t_{n+1} \equiv T$ and let $J_{0}=\left[0, t_{1}\right]$ and for $k=1,2, \ldots, n$, let $J_{k}=\left(t_{k}, t_{k+1}\right]$. Define

$$
P C=\left\{y:[0, T] \rightarrow \mathbb{R} \mid y \in C_{r d}\left(J_{k}\right), y\left(t_{k}^{ \pm}\right) \text {exist and } y\left(t_{k}^{-}\right)=y\left(t_{k}\right), k=1, \ldots, n\right\},
$$

and

$$
P C^{1}=\left\{y:[0, T] \rightarrow \mathbb{R} \mid y \in C_{r d}^{1}\left(J_{k}\right), k=1, \ldots, n\right\},
$$

where $C_{r d}\left(J_{k}\right)$ is the space of all real valued rd-continuous functions on $J_{k}$ and $C_{r d}^{1}\left(J_{k}\right)$ is the space of all rd-continuously delta-differentiable functions on $J_{k}$. The set $P C$ is a Banach space when it is endowed with the supremum norm

$$
\|u\|=\max _{0 \leq k \leq n}\left\{\|u\|_{k}\right\}
$$

where $\|u\|_{k}=\sup _{t \in J_{k}}|u(t)|$.

In this paper, we make the following conditions

(A) $a \in \mathcal{R}^{+}$is rd-continuous and $a(t)>0$ for all $t \in \mathbb{T}$.

(F) $f \in C(\mathbb{T} \times \mathbb{R}, \mathbb{R})$ and there exists a positive constant $k_{1}$ such that 


$$
|f(t, x)-f(t, y)| \leq k_{1}\|x-y\|, \text { for } x, y \in \mathbb{R} .
$$

(I) There exists a positive constant $k_{2}$ such that

$$
|I(t, x)-I(t, y)| \leq k_{2}\|x-y\|, \text { for } x, y \in \mathbb{R} .
$$

Lemma 3.1. The function $y \in P C^{1}$ is a solution of equation (1.1) if and only if $y \in P C$ is a solution of

$$
\begin{aligned}
y(t)= & \int_{0}^{t} e_{\ominus a}(t, s) a(s) H(y(s)) \Delta s \\
& +\int_{0}^{t} e_{\ominus a}(t, s) f(s, y(s)) \Delta s+\sum_{\left\{i: t_{i}<t\right\}} e_{\ominus a}\left(t, t_{i}\right) I\left(t_{i}, y\left(t_{i}\right)\right),
\end{aligned}
$$

where

$$
H(y(s))=y^{\sigma}(s)-h\left(y^{\sigma}(s)\right) .
$$

Proof. For $t \in J_{0}$, the solution of (1.1) satisfying $y(0)=0$ is

$$
y(t)=\int_{0}^{t} e_{\ominus a}(t, s) a(s) H(y(s)) \Delta s+\int_{0}^{t} e_{\ominus a}(t, 0) f(s, y(s)) \Delta s .
$$

See [6] for details. To find the solution of (1.1) on $J_{1}$ we consider the initial value problem

$$
\begin{aligned}
y^{\Delta}(t)= & -a(t) h\left(y^{\sigma}(t)\right)+f(t, y(t)), t \in J_{1}, \\
y\left(t_{1}^{+}\right)= & \int_{0}^{t_{1}} e_{\ominus a}\left(t_{1}, s\right) a(s) H(y(s)) \Delta s \\
& +\int_{0}^{t_{1}} e_{\ominus a}\left(t_{1}, s\right) f(s, y(s)) \Delta s+I\left(t_{1}, y\left(t_{1}\right)\right) .
\end{aligned}
$$

The solution to this initial value problem is

$$
\begin{aligned}
y(t)= & \int_{0}^{t} e_{\ominus a}(t, s) a(s) H(y(s)) \Delta s \\
& +\int_{0}^{t} e_{\ominus a}(t, s) f(s, y(s)) \Delta s+e_{\ominus a}\left(t, t_{1}\right) I\left(t_{1}, y\left(t_{1}\right)\right) .
\end{aligned}
$$

We proceed inductively to obtain that if $y \in P C^{1}$ is a solution of (1.1), then $y \in P C$ is a solution of

$$
\begin{aligned}
y(t)= & \int_{0}^{t} e_{\ominus a}(t, s) a(s) H(y(s)) \Delta s \\
& +\int_{0}^{t} e_{\ominus a}(t, s) f(s, y(s)) \Delta s+\sum_{\left\{i: t_{i}<t\right\}} e_{\ominus a}\left(t, t_{i}\right) I\left(t_{i}, y\left(t_{i}\right)\right) .
\end{aligned}
$$

The converse statement follows trivially and the proof is complete. 
To apply Theorem 2.6, we need to define a Banach space $\mathbb{B}$, a closed bounded convex subset $M_{L}$ of $\mathbb{B}$ and construct two mappings, one is a large contraction and the other is compact. So, we let $(\mathbb{B},\|\cdot\|)=(P C,\|\cdot\|)$ and $M_{L}=\{\varphi \in \mathbb{B}:\|\varphi\| \leq L\}$, where $L$ is positive constant. We express equation (3.3) as

$$
\varphi(t)=(B \varphi)(t)+(A \varphi)(t)=(\mathbb{C} \varphi)(t),
$$

where $A, B: M_{L} \rightarrow \mathbb{B}$ are defined by

$$
(A \varphi)(t)=\int_{0}^{t} e_{\ominus a}(t, s) f(s, \varphi(s)) \Delta s+\sum_{\left\{i: t_{i}<t\right\}} e_{\ominus a}\left(t, t_{i}\right) I\left(t_{i}, \varphi\left(t_{i}\right)\right),
$$

and

$$
(B \varphi)(t)=\int_{0}^{t} e_{\ominus a}(t, s) a(s) H(\varphi(s)) \Delta s
$$

We need the following assumptions

$$
\begin{gathered}
k_{1} L+|f(t, 0)| \leq \alpha L a(t), \\
n k_{2} L+\sum_{i=1}^{n}\left|I\left(t_{i}, 0\right)\right| \leq \beta L, \\
J(\alpha+\beta) \leq 1, \\
\max (|H(-L)|,|H(L)|) \leq \frac{2 L}{J},
\end{gathered}
$$

where $\alpha, \beta$ and $J$ are constants with $J \geq 3$.

We begin with the following proposition (see [1]) and for convenience we present, below, its proof. In the next proposition we prove that, for a well chosen function $h$, the mapping $H$ in (3.4) is a large contraction on $M_{L}$. So, let us make the following assumptions on the function $h: \mathbb{R} \rightarrow \mathbb{R}$.

(H1) $h$ is continuous on $U_{L}=[-L, L]$ and differentiable on $(-L, L)$.

(H2) $h$ is strictly increasing on $U_{L}$.

(H3) $\sup _{s \in(-L, L)} h^{\prime}(s) \leq 1$.

Proposition 3.1. Let $h: \mathbb{R} \rightarrow \mathbb{R}$ be a function satisfying (H1)-(H3). Then the mapping $H$ in (3.4) is a large contraction on the set $M_{L}$.

Proof. Let $\phi, \varphi \in M_{L}$ with $\phi^{\sigma} \neq \varphi^{\sigma}$. Then $\phi^{\sigma}(t) \neq \varphi^{\sigma}(t)$ for some $t \in \mathbb{T}$. Define the set

$$
D(\phi, \varphi)=\left\{t \in \mathbb{T}: \phi^{\sigma}(t) \neq \varphi^{\sigma}(t)\right\}
$$


Note that $\varphi^{\sigma}(t) \in U_{L}$ for all $t \in \mathbb{T}$ whenever $\varphi \in M_{L}$. Since $h$ is strictly increasing

$$
\frac{h\left(\varphi^{\sigma}(t)\right)-h\left(\phi^{\sigma}(t)\right)}{\varphi^{\sigma}(t)-\phi^{\sigma}(t)}=\frac{h\left(\phi^{\sigma}(t)\right)-h\left(\varphi^{\sigma}(t)\right)}{\phi^{\sigma}(t)-\varphi^{\sigma}(t)}>0,
$$

holds for all $t \in D(\phi, \varphi)$. On the other hand, for all $t \in D(\phi, \varphi)$, we have

$$
\begin{aligned}
|(H \phi)(t)-(H \varphi)(t)| & =\left|\phi^{\sigma}(t)-h\left(\phi^{\sigma}(t)\right)-\varphi^{\sigma}(t)+h\left(\varphi^{\sigma}(t)\right)\right| \\
& =\left|\phi^{\sigma}(t)-\varphi^{\sigma}(t)\right|\left|1-\left(\frac{h\left(\phi^{\sigma}(t)\right)-h\left(\varphi^{\sigma}(t)\right)}{\phi^{\sigma}(t)-\varphi^{\sigma}(t)}\right)\right| .
\end{aligned}
$$

For each fixed $t \in D(\phi, \varphi)$, define the set $U_{t} \subset U_{L}$ by

$$
U_{t}=\left\{\begin{array}{l}
\left(\varphi^{\sigma}(t), \phi^{\sigma}(t)\right), \text { if } \phi^{\sigma}(t)>\varphi^{\sigma}(t), \\
\left(\phi^{\sigma}(t), \varphi^{\sigma}(t)\right), \text { if } \varphi^{\sigma}(t)>\phi^{\sigma}(t),
\end{array} \quad \text { for } t \in D(\phi, \varphi)\right.
$$

The mean value theorem implies that for each fixed $t \in D(\phi, \varphi)$ there exists a real number $c_{t} \in U_{t}$ such that

$$
\frac{h\left(\phi^{\sigma}(t)\right)-h\left(\varphi^{\sigma}(t)\right)}{\phi^{\sigma}(t)-\varphi^{\sigma}(t)}=h^{\prime}\left(c_{t}\right) .
$$

By (H2) and (H3), we have

$$
1 \geq \sup _{t \in(-L, L)} h^{\prime}(t) \geq \sup _{t \in U_{t}} h^{\prime}(t) \geq h^{\prime}\left(c_{t}\right) \geq \inf _{s \in U_{t}} h^{\prime}(s) \geq \inf _{t \in(-L, L)} h^{\prime}(t) \geq 0 .
$$

Consequently, by (3.11)-(3.13), we obtain

$$
|(H \phi)(t)-(H \varphi)(t)| \leq\left|1-\inf _{u \in(-L, L)} h^{\prime}(u)\right|\left|\phi^{\sigma}(t)-\varphi^{\sigma}(t)\right|,
$$

for all $t \in D(\phi, \varphi)$. Hence, the mapping $H$ is a large contraction in the supremum norm. Indeed, fix $\epsilon \in(0,1)$ and assume that $\phi$ and $\varphi$ are two functions in $M_{L}$ satisfying

$$
\|\phi-\varphi\|=\sup _{t \in D(\phi, \varphi)}|\phi(t)-\varphi(t)| \geq \epsilon .
$$

If $\left|\phi^{\sigma}(t)-\varphi^{\sigma}(t)\right| \leq \epsilon / 2$ for some $t \in D(\phi, \varphi)$, then from (3.13) and (3.14), we get

$$
|(H \phi)(t)-(H \varphi)(t)| \leq\left|\phi^{\sigma}(t)-\varphi^{\sigma}(t)\right| \leq \frac{1}{2}\|\phi-\varphi\| .
$$

Since $h$ is continuous and strictly increasing, the function $h\left(u+\frac{\epsilon}{2}\right)-h(u)$ attains its minimum on the closed and bounded interval $[-L, L]$. Thus, if $\frac{\epsilon}{2}<\left|\phi^{\sigma}(t)-\varphi^{\sigma}(t)\right|$ for some $t \in D(\phi, \varphi)$, then from (H2) and (H3) we conclude that

$$
1 \geq \frac{h\left(\phi^{\sigma}(t)\right)-h\left(\varphi^{\sigma}(t)\right)}{\phi^{\sigma}(t)-\varphi^{\sigma}(t)}>\lambda
$$


where,

$$
\lambda=\frac{1}{2 L} \min \left\{h\left(u+\frac{\epsilon}{2}\right)-h(u), u \in[-L, L]\right\}>0 .
$$

Therefore, from (3.12), we have

$$
|(H \phi)(t)-(H \varphi)(t)| \leq(1-\lambda)\|\phi-\varphi\| .
$$

Consequently, it follows from (3.15) and (3.16) that

$$
|(H \phi)(t)-(H \varphi)(t)| \leq \eta\|\phi-\varphi\|,
$$

where

$$
\eta=\max \left\{\frac{1}{2}, 1-\lambda\right\}<1
$$

The proof is complete.

We shall prove that the mapping $\mathbb{C}$ has a fixed point which solves (1.1), whenever its derivative exists.

Lemma 3.2. For $A$ defined in (3.5), suppose that (A)-(I) and (3.7)-(3.9) hold. Then $A: M_{L} \rightarrow M_{L}$ is continuous in the supremum norm and maps $M_{L}$ into a compact subset of $M_{L}$.

Proof. We first show that $A: M_{L} \rightarrow M_{L}$. It is clear that $A: M_{L} \rightarrow P C$. In view of (3.1) and (3.2) we arrive at

$$
\begin{aligned}
|f(t, x)| & \leq|f(t, x)-f(t, 0)|+|f(t, 0)| \leq k_{1}\|x\|+|f(t, 0)| \\
|I(t, x)| & \leq|I(t, x)-I(t, 0)|+|I(t, 0)| \leq k_{2}\|x\|+|I(t, 0)| .
\end{aligned}
$$

So, for any $\varphi \in M_{L}$, we have

$$
\begin{aligned}
|(A \varphi)(t)| & \leq \int_{0}^{t} e_{\ominus a}(t, s)|f(s, \varphi(s))| \Delta s+\sum_{\left\{i: t_{i}<t\right\}} e_{\ominus a}\left(t, t_{i}\right)\left|I\left(t_{i}, \varphi\left(t_{i}\right)\right)\right| \\
& \leq \int_{0}^{t} e_{\ominus a}(t, s)\left(k_{1} L+|f(s, 0)|\right) \Delta s+\sum_{i=1}^{n} e_{\ominus a}\left(t, t_{i}\right)\left(k_{2} L+\left|I\left(t_{i}, 0\right)\right|\right), \\
& \leq \alpha L \int_{0}^{t} e_{\ominus a}(t, s) a(s) \Delta s+n k_{2} L+\sum_{i=1}^{n}\left|I\left(t_{i}, 0\right)\right| \\
& \leq \alpha L+\beta L=(\alpha+\beta) L \leq \frac{L}{J}<L .
\end{aligned}
$$

Thus, $A \varphi \in M_{L}$. Consequently, we have $A: M_{L} \rightarrow M_{L}$. 
We show that $A$ is continuous in the supremum norm. Toward this, let $\varphi, \psi \in$ $M_{L}$. Note that from $a(t)>0$ we have $\max _{s \in[0, t]}\left\{e_{\ominus a}(t, s)\right\} \leq 1$. So,

$$
\begin{aligned}
|(A \varphi)(t)-(A \psi)(t)| \leq & \int_{0}^{t} e_{\ominus a}(t, s)|f(s, \varphi(s))-f(s, \psi(s))| \Delta s \\
& +\sum_{\left\{i: t_{i}<t\right\}} e_{\ominus a}\left(t, t_{i}\right)\left|I\left(t_{i}, \varphi\left(t_{i}\right)\right)-I\left(t_{i}, \psi\left(t_{i}\right)\right)\right|, \\
\leq & k_{1} T\|\varphi-\psi\|+n k_{2}\|\varphi-\psi\| \\
\leq & \left(k_{1} T+n k_{2}\right)\|\varphi-\psi\| .
\end{aligned}
$$

Let $\epsilon>0$ be arbitrary. Define $\theta=\epsilon / K$ with $K=k_{1} T+n k_{2}$, where $k_{1}$ and $k_{2}$ are given by (3.1) and (3.2). Then, for $\|\varphi-\psi\|<\theta$ we obtain

$$
\|A \varphi-A \psi\| \leq K\|\varphi-\psi\|<\epsilon .
$$

This proves that $A$ is continuous.

It remains to show that $A$ is compact. Let $\varphi_{n} \in M_{L}$, where $n$ is a positive integer and let

$$
\gamma=\max _{t \in[0, T]}\{a(t)\}, \mu=\max _{t \in[0, T]}|f(t, 0)| .
$$

Then, as above, we see that

$$
\left\|A \varphi_{n}\right\| \leq L .
$$

Moreover, a direct calculation shows that

$$
\left(A \varphi_{n}\right)^{\triangle}(t)=-a(t)\left(A \varphi_{n}\right)(t)+f\left(t, \varphi_{n}(t)\right) .
$$

By invoking (3.1) and (3.17) we obtain

$$
\left|\left(A \varphi_{n}\right)^{\triangle}(t)\right| \leq\left(\gamma+k_{1}\right) L+\mu \leq D,
$$

for some positive constant $D$. Hence the sequence $\left(A \varphi_{n}\right)$ is uniformly bounded and equicontinuous. The Ascoli-Arzela theorem implies that a subsequence $\left(A \varphi_{n_{k}}\right)$ of $\left(A \varphi_{n}\right)$ converges uniformly. Thus $A$ is continuous and $A M_{L}$ is contained in a compact subset of $M_{L}$.

Lemma 3.3. Let $B$ be defined by (3.6) and that (H1)-(H3) and (3.10) hold. Then $B: M_{L} \rightarrow M_{L}$ is a large contraction.

Proof. We first show that $B: M_{L} \rightarrow M_{L}$. Clearly, $B: M_{L} \rightarrow P C$. So, for any $\varphi \in M_{L}$, we get by (3.6) that

$$
\begin{aligned}
|(B \varphi)(t)| & \leq \int_{0}^{t} e_{\ominus a}(t, s) a(s)|H(\varphi(s))| \triangle s \\
& \leq \max (|H(-L)|,|H(L)|) \int_{0}^{t} e_{\ominus a}(t, s) a(s) \triangle s \\
& \leq \frac{2 L}{J}<L .
\end{aligned}
$$


Thus $B \varphi \in M_{L}$. Consequently, we have $B: M_{L} \rightarrow M_{L}$.

It remains to show that $B$ is large contraction with a unique fixed point in $M_{L}$. Form the proof of Proposition 3.1 we have for $\phi, \varphi \in M_{L}$, with $\phi \neq \varphi$

$$
\begin{aligned}
|(B \phi)(t)-(B \varphi)(t)| & \leq \int_{0}^{t} e_{\ominus a}(t, s) a(s)|H(\phi(s))-H(\varphi(s))| \triangle s \\
& \leq\|\phi-\varphi\| \int_{0}^{t} e_{\ominus a}(t, s) a(s) \triangle s \\
& \leq\|\phi-\varphi\| .
\end{aligned}
$$

Then $\|B \phi-B \varphi\| \leq\|\phi-\varphi\|$. Now, let $\epsilon \in(0,1)$ be given and let $\phi, \varphi \in M_{L}$ with $\|\phi-\varphi\| \geq \epsilon$. From the proof of Proposition 3.1, we have found a $\eta<1$, such that

$$
\begin{aligned}
|(B \varphi)(t)-(B \psi)(t)| & \leq \int_{0}^{t} e_{\ominus a}(t, s) a(s)|H(\phi(s))-H(\varphi(s))| \triangle s \\
& \leq \eta\|\phi-\varphi\| \int_{0}^{t} e_{\ominus a}(t, s) a(s) \triangle s \\
& \leq \eta\|\varphi-\psi\| .
\end{aligned}
$$

Then $\|B \phi-B \varphi\| \leq \eta\|\varphi-\psi\|$. Consequently, $B$ is a large contraction on $M_{L}$.

Theorem 3.1. Let $M_{L}=\{\varphi \in P C:\|\varphi\| \leq L\}$, where $L$ is a positive constant. Suppose (A)-(I), (H1)-(H3) and (3.7)-(3.10) hold. Then equation (1.1) has a solution $\varphi$ in the subset $M_{L}$.

Proof. By Lemma 3.2, $A: M_{L} \rightarrow M_{L}$ is continuous and $A M_{L}$ is contained in a compact set. Also, from Lemma 3.3, the mapping $B: M_{L} \rightarrow M_{L}$ is a large contraction. Next, note that if $\phi, \varphi \in M_{L}$, we have

$$
\|A \phi+B \varphi\| \leq\|A \phi\|+\|B \varphi\| \leq \frac{L}{J}+\frac{2 L}{J} \leq L .
$$

Thus $A \phi+B \varphi \in M_{L}$.

Clearly, all the hypotheses of the Krasnoselskii-Burton's theorem (Theorem 2.6) are satisfied. Thus there exists a fixed point $\varphi \in M_{L}$ such that $\varphi=A \varphi+B \varphi$. Hence the equation (1.1) has a solution in $M_{L}$.

Acknowledgment. The authors would like to thank the anonymous referee for his valuable comments.

\section{REF E R E N C ES}

1. M. Adivar And Y. N. RAfFoul: Existence of periodic solutions in totally nonlinear delay dynamic equations. Electronic Journal of Qualitative Theory of Differential Equations 2009 (2009), No. 1, 1-20. 
2. A. ARdjouni AND A. Djoudi: Existence of periodic solutions for nonlinear neutral dynamic equations with functional delay on a time scale. Acta Univ. Palacki. Olomnc., Fac. rer. nat., Mathematica 52, 1 (2013) 5-19.

3. A. Ardjouni ANd A. DJoudi: Existence of periodic solutions for nonlinear neutral dynamic equations with variable delay on a time scale. Commun Nonlinear Sci Numer Simulat 17 (2012) 3061-3069.

4. A. Ardjouni And A. DJoudi: Periodic solutions in totally nonlinear dynamic equations with functional delay on a time scale. Rend. Sem. Mat. Univ. Politec. Torino Vol. 68, 4(2010), 349-359.

5. D. D. Bainov And P. S. Simeonov: Systems with Impulse Effect: Stability, Theory and Applications. Ellis Horwood Series: Mathematics and its Applications, Ellis Horwood, 1989.

6. M. Bohner And A. Peterson: Dynamic Equations on Time Scales, An Introduction with Applications. Birkhäuser, Boston, 2001.

7. M. Bohner And A. Peterson: Advances in Dynamic Equations on Time Scales. Birkhäuser, Boston, 2003.

8. T. A. Burton: Liapunov functionals, fixed points and stability by Krasnoselskii's theorem. Nonlinear Stud. 9 (2002), No. 2, 181-190.

9. T. A. Burton: Stability by Fixed Point Theory for Functional Differential Equations. Dover, New York, 2006.

10. H. Deham And A. DJoudi: Periodic solutions for nonlinear differential equation with functional delay. Georgian Mathematical Journal 15 (2008), No. 4, 635-642.

11. H. Deham AND A. DJoudi: Existence of periodic solutions for neutral nonlinear differential equations with variable delay. Electronic Journal of Differential Equations, Vol. 2010 (2010), No. 127, pp. 1-8.

12. S. Hilger: Ein Masskettenkalkül mit Anwendung auf Zentrumsmanningfaltigkeiten. PhD thesis, Universität Würzburg, 1988.

13. E. R. Kaufmann, N. Kosmatov and Y. N. Raffoul: Impulsive dynamic equations on a time scale. Electronic Journal of Differential Equations 2008 (2008), No. 67, 1-9.

14. E. R. Kaufmann And Y. N. Raffoul: Periodic solutions for a neutral nonlinear dynamical equation on a time scale. J. Math. Anal. Appl., 319 (2006), No. 1, 315325 .

15. E. R. Kaufmann And Y. N. Raffoul: Periodicity and stability in neutral nonlinear dynamic equation with functional delay on a time scale. Electronic Journal of Differential Equations 2007 (2007), No. 27, 1-12.

16. V. Lakshmikantham, D. D. Bainov, and P. S. Simeonov: Theory of Impulsive Differential Equations. Series in Modern Applied Mathematics, 6, World Scientific, New Jersey, 1994.

17. A. M. Samollenko And N. A. Perestyuk: Impulsive Differential Equations. World Scientific Seriess on Nonlinear Science, Series A: Monographs and Treatises, 14, World Scientific, New Jersey, 1995.

18. D. R. Smart: Fixed point theorems. Cambridge Tracts in Mathematics, No. 66. Cambridge University Press, London-New York, 1974. 
19. E. YANKSON: Existence of periodic solutions for totally nonlinear neutral differential equations with functional delay. Opuscula Mathematica, VoL. 32, No. 3, 2012, 617-627.

Abdelouaheb Ardjouni

Faculty of Sciences and Technology

Department of Mathematics and Informatics

University of Souk-Ahras

P.O. Box 1553

Souk-Ahras, 41000, Algeria

abd_ardjouni@yahoo.fr

Ahcene Djoudi

Faculty of Sciences, Department of Mathematics

University of Annaba

P.O. Box 12

Annaba 23000, Algeria

adjoudi@yahoo.com 